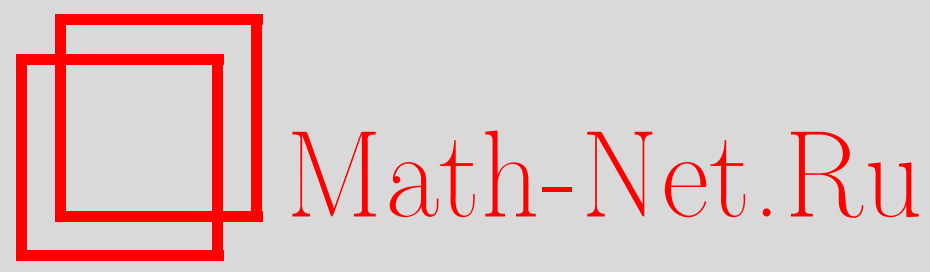

В. С. Самовол, Гладкая эквивалентность дифференциальных уравнений и линейные автоморфизмы, Матем. заметкu, 1999, том 66, выпуск 4, 567-578

DOI: https://doi.org/10.4213/mzm1199

Использование Общероссийского математического портала Math-Net.Ru подразумевает, что вы прочитали и согласны с пользовательским соглашением http://www . mathnet.ru/rus/agreement

Параметры загрузки:

IP : 3.85 .7 .115

26 апреля 2023 г., 16:45:03

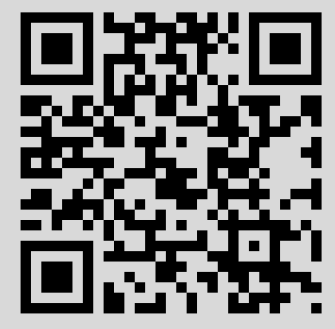




\section{ГЛАДКАЯ ЭКВИВАЛЕНТНОСТЬ ДИФФЕРЕНЦИАЛЬНЫХ УРАВНЕНИЙ И ЛИНЕЙНЫЕ АВТОМОРФИЗМЫ}

\section{В. С. Самовол}

В статье рассматриваются задачи локальной гладкой эквивалентности и линеаризации систем обькновенных дифференциальных уравнений с сохранением имеющихся у этих систем линейных автоморфизмов.

Библиография: 7 названий.

Введение. Поводом к изучению задачи, лежащей в основе данной работы, послужил доклад А. Л. Афендикова, сделанньй на научно-исследовательском семинаре под руководством А. Д. Брюно в ИМП им. М. В. Келдьша [1]. Автор доклада рассматривал систему обыкновенных дифференциальных уравнений, с которой тесно связно известное в гидродинамике уравнение Гинзбурга-Ландау. Особенность исследовавшейся системы заключается, в частности, в том, что она обладает линейным автоморфизмом, т.е. ее правая часть не меняется в результате некоторой линейной замены переменных.

Одним из наиболее эффективных методов анализа дифференциальных уравнений является их упрощение путем подходящего преобразования координат. В связи с этим представляет интерес вопрос о сохранении имеющегося автоморфизма после таких преобразований. Для формальных и аналитических систем этот вопрос исследован в [2]. В данной работе эта задача изучается для гладких систем.

Рассмотрим вещественную систему обыкновенньх дифференциальных уравнений

$$
\xi=Q(\xi)
$$

где $\xi, Q(\xi)$ - векторы из $\mathbb{R}^{n}, Q(\xi)$ - функция класса $C^{l}$ в некоторой окрестности нуля (здесь $l>0$ - целое число или $\infty), Q(0)=0$, матрица $A=Q^{\prime}(0)$ не имеет собственных чисел с нулевой действительной частью. Точка $\xi=0$ является неподвижной точкой для системы (1).

Пусть при линейном невырожденном преобразовании

$$
\xi=B \eta
$$

система (1) переходит в систему

где $\widetilde{Q}(\eta)=B^{-1} Q(B \eta)$.

$$
\dot{\eta}=\widetilde{Q}(\eta)
$$

Если при этом оказывается, что $\widetilde{Q}(\eta) \equiv Q(\eta)$, то преобразование $(2)$ называется линейньм автоморфизмом системы (1) (см. [2]). В дальнейшем предполагается наличие у системы (1) линейного автоморфизма (2). Ниже для краткости слово линейньй мы будем часто опускать. Матрицу $B$ будем назьвать матрицей автоморфизма.

Работа выполнена при финансовой поддержке Российского фонда фундаментальных исследова-
ний.
567 
ЗАмЕчАниЕ 1. В [2] понятие линейного автоморфизмавключает в себя кроме преобразования (2) и линейную замену времени $t=\mu \tau$. Это означает вьполнение равенства $\mu B^{-1} A B=A$, где $A=Q^{\prime}(0)$. Приравнивая определители левой и правой части данного равенства, для вешественной системы (1) получаем, что $\mu= \pm 1$. При этом $\mu=-1$ возможно лишь в случае, когда матрица $A$ имеет четную размерность и число ее жордановых клеток нечетной размерности четно. Ниже мы ограничимся рассмотрением случая $\mu=1$.

1. Инвариантные многообразия. Хорошо известно, что у системы (1) имеются инвариантные многообразия: устойчивое и неустойчивое, размерности которых совпадают с числом соответствуюших элементов спектра матрицы линейной части системы. Широко распространенным и весьма полезньп приемом является "вьпрямление" этих многообразий, т.е. такая замена переменных, после которой они превращаются в координатные подпространства. В нашем случае необходимо позаботиться о том, чтобы при такой замене переменных сохранялся автоморфизм системы. Покажем, что это всегда можно обеспечить.

После соответствующего линейного преобразования $\xi=P x, x=\left(x^{1}, x^{2}\right)$, нашу систему можно записать в виде

$$
\dot{x}^{1}=A_{1} x^{1}+f_{1}\left(x^{1}, x^{2}\right), \quad \dot{x}^{2}=A_{2} x^{2}+f_{2}\left(x^{1}, x^{2}\right),
$$

где $A_{1}$ и $A_{2}$ - матрицы, спектры которых расположены соответственно слева и справа от мнимой оси. Матрица $\widetilde{B}$ автоморфизма системы $(3)$ имеет вид $\widetilde{B}=P^{-1} B P$. Кроме того, ясно, что матрища $\widetilde{B}$ имеет блочно-диагональную структуру $\widetilde{B}=\operatorname{diag}\left[B_{1}, B_{2}\right]$, и справедливы равенства

$$
\begin{array}{cl}
B_{1}^{-1} A_{1} B_{1}=A_{1}, & B_{2}^{-1} A_{2} B_{2}=A_{2}, \\
f_{1}\left(B_{1} x^{1}, B_{2} x^{2}\right)=B_{1} f_{1}\left(x^{1}, x^{2}\right), & f_{2}\left(B_{1} x^{1}, B_{2} x^{2}\right)=B_{2} f_{2}\left(x^{1}, x^{2}\right) .
\end{array}
$$

У системы (3) имеются два инвариантных многообразия: устойчивое $\left(x^{2}=\varphi\left(x^{1}\right)\right)$ и неустойчивое $\left(x^{1}=\widetilde{\varphi}\left(x^{2}\right)\right)$, содержащие начало координат. Наша цель - доказать следующую теорему.

ТЕОРемА 1. Устойчивое и неустойчивое многообразия обладают автоморфизмом системы, т.е.

$$
\varphi\left(B_{1} x^{1}\right)=B_{2} \varphi\left(x^{1}\right), \quad \widetilde{\varphi}\left(B_{2} x^{2}\right)=B_{1} \widetilde{\varphi}\left(x^{2}\right) .
$$

Это, по сути дела, означает, что “вьпрямляющая” замена переменных сохраняет автоморфизм системы.

ДокАЗАТЕЛЬСТво. Ограничимся рассмотрением устойчивого многообразия. $\Phi$ ункция $\varphi(u)$ удовлетворяет уравнению в частных производных

$$
\varphi_{u}^{\prime}(u)\left(A_{1} u+f_{1}(u, \varphi(u))\right)=A_{2} \varphi(u)+f_{2}(u, \varphi(u)) .
$$

Здесь $\varphi_{u}^{\prime}(u)$ - матрица первых производных функции $\varphi(u)$.

Рассмотрим функцию $\psi(u)=B_{2}^{-1} \varphi\left(B_{1} u\right)$. Для того чтобы доказать наше утверждение, достаточно показать, что функция $\psi(u)$ удовлетворяет уравнению

$$
\psi_{u}^{\prime}(u)\left(A_{1} u+f_{1}(u, \psi(u))\right)=A_{2} \psi(u)+f_{2}(u, \psi(u)) .
$$


Тогда из единственности устойчивого многообразия следует, что $\psi(u) \equiv \varphi(u)$ и равенство (4) доказано. Покажем справедливость (6).

Из определения функции $\psi(u)$ следует, что левая часть уравнения (6) преобразуется следующим образом:

$$
\begin{aligned}
\psi_{u}^{\prime}(u)\left(A_{1} u+f_{1}(u, \psi(u))\right) & =B_{2}^{-1} \varphi_{u}^{\prime}\left(B_{1} u\right) B_{1}\left(A_{1} u+f_{1}\left(u, B_{2}^{-1} \varphi\left(B_{1} u\right)\right)\right) \\
& =B_{2}^{-1} \varphi_{u}^{\prime}\left(B_{1} u\right)\left(B_{1} A_{1} u+B_{1} f_{1}\left(u, B_{2}^{-1} \varphi\left(B_{1} u\right)\right)\right) \\
& =B_{2}^{-1} \varphi_{u}^{\prime}\left(B_{1} u\right)\left(A_{1} B_{1} u+f_{1}\left(B_{1} u, B_{2} B_{2}^{-1} \varphi\left(B_{1} u\right)\right)\right) \\
& =B_{2}^{-1} \varphi_{u}^{\prime}\left(B_{1} u\right)\left(A_{1} B_{1} u+f_{1}\left(B_{1} u, \varphi\left(B_{1} u\right)\right)\right) .
\end{aligned}
$$

Далее используем то, что функция $\varphi(u)$ удовлетворяет уравнению (5), которое мы запишем таким образом:

$$
\varphi_{u}^{\prime}\left(B_{1} u\right)\left(A_{1} B_{1} u+f_{1}\left(B_{1} u, \varphi\left(B_{1} u\right)\right)\right)=A_{2} \varphi\left(B_{1} u\right)+f_{2}\left(B_{1} u, \varphi\left(B_{1} u\right)\right)
$$

Отсюда и из (7) получаем следующее:

$$
\begin{aligned}
\psi_{u}^{\prime}(u)\left(A_{1} u+f_{1}(u, \psi(u))\right) & =B_{2}^{-1} A_{2} \varphi\left(B_{1} u\right)+B_{2}^{-1} f_{2}\left(B_{1} u, \varphi\left(B_{1} u\right)\right) \\
& =A_{2} B_{2}^{-1} \varphi\left(B_{1} u\right)+f_{2}\left(u, B_{2}^{-1} \varphi\left(B_{1} u\right)\right) \\
& =A_{2} \psi(u)+f_{2}(u, \psi(u))
\end{aligned}
$$

и равенство (6) установлено.

Отметим, что использованное вьше равенство

$$
B_{2}^{-1} f_{2}\left(B_{1} u, \varphi\left(B_{1} u\right)\right)=f_{2}\left(u, B_{2}^{-1} \varphi\left(B_{1} u\right)\right)
$$

следует из того, что $\widetilde{B}$ является матрицей автоморфизма системы (3). Теорема доказана.

Отметим, что если $\xi\left(\xi^{0}, t\right)$ - решение системы (1), удовлетворяющее начальному условию $\xi\left(\xi^{0}, 0\right)=\xi^{0}$ и $(2)$ - автоморфизм системы, то имеют место равенства

$$
\xi\left(B \xi^{0}, t\right)=B \xi\left(\xi^{0}, t\right), \quad \xi\left(B^{-1} \xi^{0}, t\right)=B^{-1} \xi\left(\xi^{0}, t\right) .
$$

Действительно, поскольку (2) является автоморфизмом системы (1), то вместе с $\xi\left(\xi^{0}, t\right)$ решением этой системы является и функция $\xi_{1}\left(\xi^{0}, t\right)=B^{-1} \xi\left(B \xi^{0}, t\right)$, причем $\xi_{1}\left(\xi^{0}, 0\right)=\xi^{0}$. Отсюда и из теоремы единственности, очевидно, следуют равенства (8). Таким образом, оператор сдвига по траекториям системы обладает тем же автоморфизмом, что и сама система.

2. Гладкая эквивалентность систем уравнений. Следующий вопрос, которьй нас интересует, заключается в анализе ситуации, описьваемой известной теоремой Стернберга-Ченя о локальной гладкой эквивалентности систем обыкновенньх дифференциальных уравнений [3, теорема 12.2]. Достаточные условия такой эквивалентности и методы построения сопрягаюшего диффеоморфизма хорошо известны. Остается выяснить возможность сохранения автоморфизма системы уравнений при соответствующих ее преобразованиях. 
Рассмотрим две системы уравнений

$$
\begin{aligned}
& \dot{\xi}=Q_{1}(\xi), \\
& \dot{\eta}=Q_{2}(\eta)
\end{aligned}
$$

где $\xi, \eta, Q_{1}, Q_{2} \in \mathbb{R}^{n}, n>0$, функции $Q_{1}, Q_{2}$ принадлежат классу $C^{l}, l \geqslant 1$,

$$
Q_{1}(0)=Q_{2}(0)=0, \quad \frac{\partial Q_{1}(0)}{\partial \xi}=\frac{\partial Q_{2}(0)}{\partial \eta}=A=\operatorname{diag}\left[A_{1}, A_{2}\right]
$$

$A_{i}$ - квадратные матрицы размера $n_{i}, n_{1}+n_{2}=n$, спектр матрицы $A_{1}$ лежит левее, а спектр матрицы $A_{2}$ - правее мнимой оси.

Пусть, кроме того, системы (9) и (10) обладают линейным автоморфизмом с матрицей $B$, т.е.

$$
B^{-1} Q_{1}(B \xi) \equiv Q_{1}(\xi), \quad B^{-1} Q_{2}(B \eta) \equiv Q_{2}(\eta)
$$

В соответствии с теоремой 1 будем считать, что у систем (9) и (10) имеются "выпрямленные" инвариантные многообразия

$$
\begin{array}{ll}
M_{1 \xi}=\left\{\xi_{i}=0,1 \leqslant i \leqslant n_{1}\right\}, & M_{2 \xi}=\left\{\xi_{i}=0, n_{1}+1 \leqslant i \leqslant n_{1}+n_{2}\right\} \\
M_{1 \eta}=\left\{\eta_{i}=0,1 \leqslant i \leqslant n_{1}\right\}, & M_{2 \eta}=\left\{\eta_{i}=0, n_{1}+1 \leqslant i \leqslant n_{1}+n_{2}\right\} .
\end{array}
$$

Через $\Lambda=\left\{\lambda_{i}, 1 \leqslant i \leqslant n\right\}$ будем обозначать спектр матрищы $A$.

Если существует диффеоморфизм $\xi=g(\eta)$ класса $C^{k}, k \geqslant 1$, приводящий систему (9) к виду (10) в некоторой окрестности начала координат, то говорят, что системы (9) и (10) $C^{k}$-эквивалентны (локально эквивалентны). Если при этом система (10) линейна, то говорят о $C^{k}$-линеаризации системы (9). Нас интересует существование указанного диффеоморфизма, коммутирующего с линейным автоморфизмом системы, т.е. такого, что $g(B \eta) \equiv B g(\eta)$. В этом случае мы будем говорить о $C^{k}$-эквивалентности систем (9) и (10) с сохранением автоморфизма.

Возвращаясь к уравнению Гинзбурга-Ландау, упомянутому в начале данной статьи, и связанной с ним системе уравнений (1), обладающей автоморфизмом (2), отметим, что матрица такого автоморфизма удовлетворяет условию

$$
B^{m}=E,
$$

где $E$ - единичная матрища, а $m>0$ - некоторое целое число. Это означает, что собственные числа матрицы $B$ являются корнями степени $m$ из единицы, а также, что жорданова форма матрицы $B$ является диагональной матрицей.

ТЕОрема 2. Для систем (9) $u(10)$, әде $l=k+1$ и имеет место равенство (11), существует иелое число $N=N(k, \Lambda)$, обладающее следующим свойством: если при малых $\xi$ для всех частных производных $G^{(i)}(\xi)$ порядка $i \leqslant k$ разности $G(\xi)=Q_{1}(\xi)-Q_{2}(\xi)$ справедлива оченка $\left\|G^{(i)}(\xi)\right\| \leqslant D\left\|\left(\xi_{1}, \ldots, \xi_{n}\right)\right\|^{N-i}(D-$ некоторая константа), то для системы (9) существует невырожденная замена переменных класса $C^{k}$

$$
\xi=g(\eta)
$$


которая удовлетворяет условию $g(B \eta)=B g(\eta)$ и приводит эту систему к ви$\partial y(10)$, т.е. системы (9) и (10) $C^{k}$-әквивалентны с сохранением автоморфизма.

Существует несколько способов построения сопрягающего диффеоморфизма. Для того чтобы такой диффеоморфизм коммутировал с автоморфизмом системы, воспользуемся схемой, предложенной в доказательстве теоремы 1 из [4].

Введем обозначения $\xi=(x, y), x=\left(\xi_{1}, \ldots, \xi_{n_{1}}\right), y=\left(\xi_{n_{1}+1}, \ldots, \xi_{n_{1}+n_{2}}\right), r_{1}=\|x\|$, $r_{2}=\|y\|, r_{3}=\|(x, y)\|$.

Если $\alpha=\left(\alpha_{1}, \ldots, \alpha_{n}\right)$ - набор из неотрицательных целых чисел, то через $F^{\alpha}$ будем обозначать производную

$$
F^{\alpha}=\frac{\partial^{|\alpha|} F(\xi)}{\partial \xi_{1}^{\alpha_{1}} \cdots \partial \xi_{n}^{\alpha_{n}}}, \quad|\alpha|=\sum_{i=1}^{n} \alpha_{i} .
$$

В дальнейшем, если возникнет необходимость показать ограниченность какой-либо величины произвольной константой, будем пользоваться так назьваемой "универсальной" константой $D>0$, считая, что $D+D=D, D \cdot D=D$.

В дальнейшем нам понадобится следующее вспомогательное утверждение, аналогичное лемме 1 из [4].

ЛЕмма 1. Если при мальх $\|\xi\| F(\xi) \in C^{p}$,

$$
\left|F^{\alpha}\right| \leqslant D r_{3}^{N-|\alpha|}, \quad N \geqslant 2(p+1), \quad 0 \leqslant|\alpha| \leqslant p,
$$

то существуют функиии $F_{i}(\xi), i=1,2$, такие, что $F(\xi)=F_{1}(\xi)+F_{2}(\xi), F_{i}(\xi) \in C^{p}$, $i=1,2$, и при мальх $\|\xi\|$

$$
\left|F_{i}^{\alpha}\right| \leqslant D r_{i}^{N_{1}-|\alpha|}, \quad 0 \leqslant|\alpha| \leqslant p, \quad i=1,2,
$$

$N_{1}=[(N+1) / 2]-$ иелая часть числа $(N+1) / 2$.

Если функция $F(\xi)$ обладает автоморфизмом системы, удовлетворяющим условию (11), то әтим же автоморфизмом обладают и функиии $F_{i}(\xi)$, т.е.

$$
F_{i}(B \xi)=B F_{i}(\xi), \quad i=1,2 .
$$

ДокАЗАТЕльство. Из неравенства (12) следует справедливость неравенства

$$
\begin{gathered}
\left|F^{\alpha}\right| \leqslant D\left(h_{1}+h_{2}\right)^{N-|\alpha|}, \quad N \geqslant 2(p+1), \quad 0 \leqslant|\alpha| \leqslant p, \\
h_{1}=h_{1}(x)=\|x\|+\left\|B_{1} x\right\|+\left\|B_{1}^{2} x\right\|+\cdots+\left\|B_{1}^{m-1} x\right\|, \\
h_{2}=h_{2}(y)=\|y\|+\left\|B_{2} y\right\|+\left\|B_{2}^{2} y\right\|+\cdots+\left\|B_{2}^{m-1} y\right\| .
\end{gathered}
$$

Из (14) при $|\alpha|=0$ следует, что функцию $F(\xi)$ можно представить в виде $F(\xi)=$ $\left(h_{1}+h_{2}\right)^{N} \varphi(\xi)$, где $\varphi(\xi)$ - ограниченная функция, принадлежащая классу $C^{p}$ всюду за исключением начала координат. Разлагая $\left(h_{1}+h_{2}\right)^{N}$ по биному Ньютона, получаем, что $F(\xi)=\left(h_{1}^{N_{1}} u_{1}\left(h_{1}, h_{2}\right)+h_{2}^{N_{1}} u_{2}\left(h_{1}, h_{2}\right)\right) \varphi(\xi)$, где $u_{i}\left(h_{1}, h_{2}\right)$ - многочлены от своих переменных, $i=1,2, N_{1}=[(N+1) / 2]$.

Положим $F_{i}(z)=h_{i}^{N_{1}} u_{i}\left(h_{1}, h_{2}\right) \varphi(z), i=1,2$. Повторяя рассуждения доказательства леммы 1 из [4], получаем, что (13) выполняется при малых $\|\xi\|$. Вследствие условия (11) из построения функций $h_{i}(\xi)$ получаем, что $h_{1}\left(B_{1} x\right) \equiv h_{1}(x), h_{2}\left(B_{2} y\right) \equiv h_{2}(y)$. Отсюда следует справедливость равенств $u(B \xi) \equiv u(\xi), v(B \xi) \equiv v(\xi)$.

Поскольку $F(\xi)=(u(\xi)+v(\xi)) \varphi(\xi), F(B \xi)=B F(\xi)$ и $u(\xi)+v(\xi)=\left(h_{1}+h_{2}\right)^{N}>0$ при $\xi \neq 0$, то $\varphi(B \xi)=B \varphi(\xi)$. Следовательно, $F_{i}(B \xi)=B F_{i}(\xi), i=1,2$.

Из последнего равенства с учетом (13) получаем, что все требования леммы вьполнены. Лемма доказана. 
ДОКАЗАТЕЛЬСТВО ТЕОРЕМЫ 2. В соответствии с теоремой 1 будем считать, что у систем (9) и (10) имеются “вьпрямленные" инвариантные многообразия

$$
\begin{array}{ll}
M_{1 \xi}=\left\{\xi_{i}=0,1 \leqslant i \leqslant n_{1}\right\}, & M_{2 \xi}=\left\{\xi_{i}=0, n_{1}+1 \leqslant i \leqslant n_{1}+n_{2}\right\} \\
M_{1 \eta}=\left\{\eta_{i}=0,1 \leqslant i \leqslant n_{1}\right\}, & M_{2 \eta}=\left\{\eta_{i}=0, n_{1}+1 \leqslant i \leqslant n_{1}+n_{2}\right\} .
\end{array}
$$

Введем обозначения $\xi=(x, y), x=\left(\xi_{1}, \ldots, \xi_{n_{1}}\right), y=\left(\xi_{n_{1}+1}, \ldots, \xi_{n_{1}+n_{2}}\right), \eta=(u, v)$, $u=\left(\eta_{1}, \ldots, \eta_{n_{1}}\right), v=\left(\eta_{n_{1}+1}, \ldots, \eta_{n_{1}+n_{2}}\right)$.

Согласно доказанной лемме 1 функцию $Q(\xi)=Q_{1}(\xi)-Q_{2}(\xi)$ можно представить в виде $Q(\xi)=R(\xi)+P(\xi)$, где $R(\xi), P(\xi) \in C^{k+1}, R(B \xi)=B R(\xi), P(B \xi)=B P(\xi)$, a также

$$
\left\|R^{\alpha}\right\| \leqslant D\|x\|^{N_{1}-|\alpha|}, \quad\left\|P^{\alpha}\right\| \leqslant D\|y\|^{N_{1}-|\alpha|}, \quad 0 \leqslant|\alpha| \leqslant k, \quad N_{1}=\left[\frac{N+1}{2}\right] .
$$

Перепишем систему (9) в виде

$$
\dot{\xi}=Q_{2}(\xi)+R(\xi)+P(\xi) .
$$

Рассмотрим также систему

$$
\dot{\zeta}=Q_{2}(\zeta)+R(\zeta), \quad \zeta=\left(x^{1}, y^{1}\right), \quad x^{1} \in \mathbb{R}^{n_{1}}, \quad y^{1} \in \mathbb{R}^{n_{2}}
$$

Очевидно, система (16) обладает автоморфизмом (2) по построению.

Мы покажем, что при $N_{1}>(2 k+1) \lambda_{\max } / \lambda_{\min }$, где

$$
\lambda_{\max }=\max _{1 \leqslant i \leqslant n}\left|\operatorname{Re} \lambda_{i}\right|, \quad \lambda_{\min }=\min _{1 \leqslant i \leqslant n}\left|\operatorname{Re} \lambda_{i}\right|,
$$

системы (10) и (16) $C^{k}$-эквивалентны, при этом сопрягающее преобразование коммутирует с автоморфизмом (2). Аналогично показывается $C^{k}$-эквивалентность систем (9) и (16) с сохранением автоморфизма. Из транзитивности свойства $C^{k}$-эквивалентности следует $C^{k}$-эквивалентность систем $(9)$ и (10), что и утверждает теорема.

Отметим, что у системы (16) многообразия $\left\{x^{1}=0\right\},\left\{y^{1}=0\right\}$ являются инвариантными, что следует из инвариантности многообразий $M_{1 \eta}, M_{2 \eta}$ и оценок $(15)$ при $|\alpha|=0$.

Пусть $\eta\left(\eta^{0}, t\right), \zeta\left(\zeta^{0}, t\right)$ являются решениями систем $(10)$ и $(16)$ с начальными условиями $\eta\left(\eta^{0}, 0\right)=\eta^{0}, \zeta\left(\zeta^{0}, 0\right)=\zeta^{0}$. Рассмотрим построенноев [4] отображение, переводящее траектории систем (10) и (16) друг в друга:

$$
\begin{aligned}
\eta_{i}^{0} & =H_{i}\left(\zeta^{0}\right), \quad 1 \leqslant i \leqslant n, \\
H_{i}\left(\zeta^{0}\right) & =\zeta_{i}^{0}+\int_{0}^{+\infty} H_{i}\left(\zeta^{0}, \tau\right) d \tau, \quad 1 \leqslant i \leqslant n, \\
H_{i}\left(\zeta^{0}, \tau\right) & =\sum_{j=1}^{n} H_{i j}\left(\zeta^{0}, \tau\right), \quad 1 \leqslant i \leqslant n, \\
H_{i j}\left(\zeta^{0}, \tau\right) & =\frac{\partial \eta_{i}\left(\zeta\left(\zeta^{0}, \tau\right),-\tau\right)}{\partial \eta_{j}^{0}} R_{j}\left(\zeta\left(\zeta^{0}, \tau\right)\right), \quad 1 \leqslant i, j \leqslant n .
\end{aligned}
$$


В работе [4] установлено, что отображение (17) невырожденно и принадлежит классу $C^{k}$, если $N_{1}>(2 k+1) \lambda_{\max } / \lambda_{\min }$. Осталось показать, что оно коммутирует с автоморфизмом (2), т.е. что

$$
H\left(B \zeta^{0}\right)=B H\left(\zeta^{0}\right)
$$

Для установления справедливости равенства (18) достаточно показать, что

$$
\frac{\partial \eta\left(\zeta\left(B \zeta^{0}, \tau\right),-\tau\right)}{\partial \eta^{0}} R\left(\zeta\left(B \zeta^{0}, \tau\right)\right)=B \frac{\partial \eta\left(\zeta\left(\zeta^{0}, \tau\right),-\tau\right)}{\partial \eta^{0}} R\left(\zeta\left(\zeta^{0}, \tau\right)\right)
$$

где $\partial \eta(\zeta, \tau) / \partial \eta^{0}$ - матрица первых частных производных функции $\eta\left(\eta^{0}, \tau\right)$ при $\eta^{0}=\zeta$.

Из (8) следует, что

$$
\frac{\partial \eta\left(B \eta^{0},-\tau\right)}{\partial \eta^{0}} B=B \frac{\partial \eta\left(\eta^{0},-\tau\right)}{\partial \eta^{0}}
$$

Кроме того, из построения функции $R(\zeta)$ имеем $R\left(B \zeta\left(\zeta^{0}, \tau\right)\right)=B R\left(\zeta\left(\zeta^{0}, \tau\right)\right)$. В результате получаем

$$
\begin{aligned}
\frac{\partial \eta\left(\zeta\left(B \zeta^{0}, \tau\right),-\tau\right)}{\partial \eta^{0}} R\left(\zeta\left(B \zeta^{0}, \tau\right)\right) & =B \frac{\partial \eta\left(\zeta\left(\zeta^{0}, \tau\right),-\tau\right)}{\partial \eta^{0}} B^{-1} B R\left(\zeta\left(\zeta^{0}, \tau\right)\right) \\
& =B \frac{\partial \eta\left(\zeta\left(\zeta^{0}, \tau\right),-\tau\right)}{\partial \eta^{0}} R\left(\zeta\left(\zeta^{0}, \tau\right)\right)
\end{aligned}
$$

что и завершает доказательство теоремы. Теорема 2 доказана.

Обратим внимание на тот факт, что теорему 2 мы доказали при том предположении, что автоморфизм систем (9) и (10) удовлетворяет условию (11). Оказьвается, что если условие (11) не вьполняется, то сопрягающего диффеоморфизма для систем (9) и (10), который бы коммутировал с автоморфизмом, может не существовать. При этом правые части систем могут отличаться членами сколь угодно высокого порядка. Покажем это.

ПримеР 1. Рассмотрим две системы уравнений в $\mathbb{R}^{3}$ :

$$
\begin{gathered}
\dot{x}=x, \quad \dot{y}=-y, \quad \dot{z}=z+x^{m} y^{m-1} \\
\dot{u}=u, \quad \dot{v}=-v, \quad \dot{w}=w
\end{gathered}
$$

( $m$ - произвольное положительное целое число), обладающие автоморфизмом с матрицей

$$
B=\left(\begin{array}{ccc}
2 & 0 & 0 \\
0 & \frac{1}{2} & 0 \\
0 & 0 & 2
\end{array}\right)
$$

Покажем, что не существует невырожденного диффеоморфизма $\xi=H(\eta)$ (здесь $\xi=$ $(x, y, z), \eta=(u, v, w)$ - вектор-столбцы, записанные в строки), приводящего систему (19) к виду (20) и коммутирующего с автоморфизмом с матрищей (21), т.е. такого, что $H(B \eta)=B H(\eta)$. Доказательство проведем от обратного.

Пусть диффеоморфизм с указанными свойствами сушествует. Поскольку у системы (20) имеется инвариантное многообразие $w=0$, у системы (19) в некоторой окрестности начала координат существует инвариантное многообразие вида

$$
z=f(x, y)
$$


обладающее автоморфизмом (21), т.е. выполняется условие

$$
f(2 x, 0.5 y)=2 f(x, y) .
$$

Покажем, что это невозможно.

Пусть окрестность нуля, в которой существует инвариантноемногообразие (22), имеет радиус $\varepsilon$. Рассуждая как при доказательстве теоремы 1 в работе [5], можно показать, что функция $f(x, y)$ удовлетворяет функциональному уравнению

$$
f\left(\frac{x y}{\delta}, \delta\right)=\frac{y}{\delta}\left(f(x, y)+x^{m} y^{m-1} \ln \left|\frac{y}{\delta}\right|\right), \quad 0<\delta<\varepsilon,
$$

которое, как нетрудно видеть, противоречит равенству (23). Следовательно, системы (19)и (20) не являются гладко эквивалентньми с сохранением автоморфизма с матрицей (21) при любом сколь угодно большом $m$. Причиной этого является, видимо, то обстоятельство, что инвариантные многообразия вида (22), которые имеются у системы (19), не являются инвариантными относительно автоморфизма системы. Данный автоморфизм переводит указанные многообразия не в себя, а в другое многообразие того же вида. Вместе с тем при линеаризации (если таковая существует) одно из таких многообразий должно переходить в координатную плоскость $w=0$, которая инвариантна относительно автоморфизма системы. Это противоречит тому, что линеаризующий диффеоморфизм коммутирует с автоморфизмом системы.

3. Гладкая линеаризация систем уравнений. Исследуем теперь проблему существования гладкого линеаризующего преобразования системы $(1)$, коммутирующего с ее автоморфизмом (2).

По-прежнему, мы будем считать, что у системы (1) устойчивое и неустойчивое многообразия "выпрямлены”, т.е. совпадают с соответствующими координатными подпространствами. Это, в частности, означает, что матрища $A$ имеет блочно-диагональную структуру $A=\operatorname{diag}\left[A_{1}, A_{2}\right]$, где собственные числа матрицы $A_{1}$ лежат левее, а собственные числа матрицы $A_{2}$ - правее мнимой оси.

В дальнейшем нам будет необходимо производить некоторые линейные преобразования координат в исследуемой системе. В частности, будет полезно приведение матрицы линейной части системы к жордановой форме. В подобных случаях в новых координатах система может уже иметь комплексньй вид. Важно отметить, что поскольку исходная система вещественна, то при этом всегда будет соблюдаться так назьваемое условие вещественности (см. [2, гл. 3, п. 1.9]). Поэтому без ограничения общности можно считать, что матрища линейной части системы (1) имеет жорданову форму, а сама система (1) и матрица $B$ автоморфизма (2) удовлетворяют условию вещественности.

Хорошо известно, что с помощью преобразования, близкого к тождественному,

$$
\xi=x+F(x), \quad\|F(x)\|=o(\|x\|)
$$

где $F(x)$ - полином от своих переменных, можно систему (1) привести к виду

$$
\dot{x}=A x+R_{1}(x)+R_{2}(x) .
$$

Здесь $R_{1}(x)$ - полином, состоящий из резонансных членов степени, не превосходящей некоторого целого числа $N$, а для функции $R_{2}(x)$ вьполняется оценка $\left\|R_{2}(x)\right\|=o\left(\|x\|^{N}\right)$ 
при $\|x\| \rightarrow 0$. При этом число $N$ можно выбрать сколь угодно большим. Кроме того, из $[2$, гл. 3, п. $1.9,1.10]$ следует, что преобразование $(24)$ можно выбрать таким, чтобы оно удовлетворяло условию вещественности, а также коммутировало с автоморфизмом системы, т.е. $F(B x)=B F(x)$. Таким образом, для функций $R_{1}(x), R_{2}(x)$ вьполняются условия

$$
R_{1}(B x)=B R_{1}(x), \quad R_{2}(B x)=B R_{2}(x) .
$$

Пусть задано некоторое целое число $k>0$. Пусть также для системы $(25)$ вьполнено неравенство $N>N(k, \Lambda)$, где $N(k, \Lambda)$ - число, существование которого утверждается в теореме 2 . Тогда, применяя к системе (25) теорему 2 , получаем, что сушествует близкое к тождественному преобразование, коммутирующее с автоморфизмом системы, приводящее эту систему к виду

$$
\dot{y}=A y+R_{1}(y) .
$$

Отметим, что из доказательства теоремы 2 следует, что указанноепреобразование удовлетворяет условию вещественности.

Для формулировки заключительных результатов запишем систему (26) в виде

$$
\dot{y}_{i}=\varepsilon_{i} y_{i-1}+\lambda_{i} y_{i}+a_{i}(y), \quad 1 \leqslant i \leqslant n,
$$

$\varepsilon_{i}=0$ или $\varepsilon_{i}=\varepsilon, \varepsilon>0, a_{i}(y)=\sum_{|s|=2}^{N} a_{s}^{i} y^{s}, s=\left(s_{1}, \ldots, s_{n}\right)$ - неотрищательные целочисленные наборы, $|s|=\sum_{i=1}^{n} s_{i}$. Кроме того, считаем, что $\operatorname{Re} \lambda_{i+1} \leqslant \operatorname{Re} \lambda_{i}<0$ при $1 \leqslant i \leqslant n_{1}-1$ и $\operatorname{Re} \lambda_{i+1} \geqslant \operatorname{Re} \lambda_{i}>0$ при $n_{1}+1 \leqslant i \leqslant n_{1}+n_{2}-1, n_{1}+n_{2}=n$.

Для каждого целочисленного набора $s \in W=\bigcup_{i=1}^{n}\left\{s: a_{s}^{i} \neq 0\right\}$ положим

$$
\begin{aligned}
\rho_{m, k}(s) & =\left\{\begin{array}{l}
\sum_{i=1}^{m} s_{i}\left|\operatorname{Re} \lambda_{i}\right|-k\left|\operatorname{Re} \lambda_{m}\right|, \quad 1 \leqslant m \leqslant n_{1}, \\
\sum_{i=n_{1}+1}^{m} s_{i} \operatorname{Re} \lambda_{i}-k \operatorname{Re} \lambda_{m}, n_{1}+1 \leqslant m \leqslant n,
\end{array}\right. \\
\rho_{k}(s) & =\max _{1 \leqslant m \leqslant n} \rho_{m, k}(s) .
\end{aligned}
$$

Будем говорить, что набор $s$ удовлетворяет условию $S(k)$, если вьполняется неравенство $\rho_{k}(s)>0$. Если же это неравенство вьполняется для каждого набора $s \in W$, то будем говорить, что система (27) удовлетворяет условию $S(k)$.

Рассмотрим вместе с системой (27) соответствующую линейную систему

$$
\dot{z}_{i}=\varepsilon_{i} z_{i-1}+\lambda_{i} z_{i}, \quad 1 \leqslant i \leqslant n .
$$

Имеет место следующее утверждение [6, теорема 3].

Если для системы (27) выполнено условие $S(k)$, то существует близкое к тождественному преобразование

$$
y_{i}=z_{i}+h_{i}(z), \quad\left|h_{i}(z)\right|=o(\|z\|), \quad 1 \leqslant i \leqslant n,
$$

класса $C^{k}$, приводящее систему (27) к виду (28), т.е. системы $(27)$ и $(28) C^{k}$-эквивалентны. Таким образом, условие $S(k)$ является достаточным условием $C^{k}$-линеаризации системы (27). Вопрос, которьй осталось обсудить, заключается в том, можно ли построить преобразование (29) так, чтобы оно коммутировало с автоморфизмом системы (27). Ответ на этот вопрос дает следующая теорема. 
ТЕОремА 3. Если матрица В автоморфизма системы (27) удовлетворяет условию (11), то условие $S(k)$ является достаточным условием для существования преобразования (29), приводящего эту систему к линейному виду (28) и коммутирующего с автоморфизмом системы, т.е. система $(27) C^{k}$-линеаризуема $c$ сохранением автоморфизма.

ДокАЗАТЕЛЬСТВО. Искомые функции $h_{i}(z)$ должны удовлетворять системе уравнений в частных производных, характеристическая система которой имеет вид

$$
\dot{z}_{i}=\varepsilon_{i} z_{i-1}+\lambda_{i} z_{i}, \quad \dot{h}_{i}=\varepsilon_{i} h_{i-1}+\lambda_{i} h_{i}+a_{i}(z+h), \quad 1 \leqslant i \leqslant n .
$$

Наша цель - показать, что у системы (30) имеется инвариантное многообразие класса $C^{k}$ вида $h=\varphi(z),\|\varphi(z)\|=o(\|z\|)$, удовлетворяющее условию $\varphi(B z)=B \varphi(z)$. Это будет означать, что отображение $y=z+\varphi(z)$ является искомым.

Следуя технологии линеаризации, разработанной в [5]-[7], произведем в системе (30) замену переменных $h=g+\Phi(z), \Phi(z) \in C^{k}$, после которой данная система примет вид

$$
\dot{z}_{i}=\varepsilon_{i} z_{i-1}+\lambda_{i} z_{i}, \quad \dot{g}_{i}=\varepsilon_{i} g_{i-1}+\lambda_{i} g_{i}+\widetilde{a}_{i}(z, g)+\widetilde{a}_{i}(z), \quad 1 \leqslant i \leqslant n,
$$

где функции $\widetilde{a}_{i}(z, g), \widetilde{a}_{i}(z)$ таковы, что $\widetilde{a}_{i}(z, 0) \equiv 0,\left|\widetilde{a}_{i}(z)\right|=o\left(\|z\|^{N}\right)$, причем число $N$ можно сделать сколь угодно большим.

Ниже мы покажем, что функция $\Phi(z)$ может быть построена так, что

$$
\Phi(B z)=B \Phi(z)
$$

Вследствие этого система (31) обладает линейным автоморфизмом с матрищей $\widetilde{B}=\operatorname{diag}[B, B]$.

Применяя к системе (31) доказанную вьше теорему 2 , получаем, что существует преобразование $g=g^{1}+\Phi^{1}(z),\left\|\Phi^{1}(z)\right\|=o(\|z\|), \Phi^{1}(z) \in C^{k}$, приводящее эту систему к виду

$$
\dot{z}_{i}=\varepsilon_{i} z_{i-1}+\lambda_{i} z_{i}, \quad \dot{g}_{i}^{1}=\varepsilon_{i} g_{i}^{1}+\lambda_{i} g_{i}^{1}+\widetilde{a}_{i}\left(z, g^{1}\right), \quad 1 \leqslant i \leqslant n .
$$

При этом для функции $\Phi^{1}(z)$ вьполняется условие $\Phi^{1}(B z)=B \Phi^{1}(z)$. У системы $(33)$ многообразие $g^{1}=0$ инвариантно, следовательно, у системы $(31)$ многообразие $g=\Phi^{1}(z)$ также инвариантно, откуда следует, что преобразование $h=\varphi(z)=\Phi(z)+\Phi^{1}(z) \in C^{k}$ является искомым и удовлетворяет требуемому условию $\varphi(B z)=B \varphi(z)$.

Нам осталось показать, что функция $\Phi(z)$ может быть построена так, что вьполнено условие (32).

В работах [5]-[7] вектор-функция $\Phi(z)=\left(\Phi_{1}(z), \ldots, \Phi_{n}(z)\right)$ строится следующим образом:

$$
\Phi_{i}(z)=\sum_{|s|=2}^{N} b_{s}^{i} z^{s} T^{\varpi}, \quad 1 \leqslant i \leqslant n,
$$

где $z^{s}$ - резонансные наборы, удовлетворяющие условию $S(k), T^{\varpi}=t_{1}^{\varpi_{1}} \cdots t_{n}^{\varpi_{n}}-$ мономы от переменных $t_{1}(z), \ldots, t_{n}(z)$, причем функции $t_{j}(z), 1 \leqslant j \leqslant n$, таковы, что производная каждой из них в силу линейной системы (28) равна 1. 
Из способа построения функций $\Phi_{i}(z)$, приведенного в [5]-[7], следует, что для вектор-функции $\widetilde{\Phi}(z)=\left(\widetilde{\Phi}_{1}(z), \ldots, \widetilde{\Phi}_{n}(z)\right)$,

$$
\widetilde{\Phi}_{i}(z)=\sum_{|s|=2}^{N} b_{s}^{i} z^{s}, \quad 1 \leqslant i \leqslant n
$$

выполняется условие

$$
\widetilde{\Phi}(B z)=B \widetilde{\Phi}(z)
$$

Ниже мы покажем, что функции $t_{j}(z), 1 \leqslant j \leqslant n$, можно выбрать таким образом, что для них будет вьполняться условие

$$
t_{j}(B z)=t_{j}(z), \quad 1 \leqslant j \leqslant n
$$

Тогда с учетом (35) для функции $\Phi(z)$ будет выполняться требуемое условие $(32)$, и теорема 3 будет доказана. Нам осталось убедиться в справедливости равенства (36).

Пусть для набора $s$ вьполнено условие $\rho_{k}(s)>0$. Рассмотрим непустое множество $M_{s}$ целых чисел $\widetilde{m}, 1 \leqslant \widetilde{m} \leqslant n$, для которых справедливо неравенство $\rho_{\widetilde{m}, k}(s)>0$, и определим число

$$
\bar{m}=m(s)=\max _{\tilde{m} \in M_{s}} \widetilde{m}
$$

являющееся максимальным элементом множества $M_{s}$.

Отметим, что если $\bar{m}=m(s)<n$, то $\operatorname{Re} \lambda_{\bar{m}+1} \neq \operatorname{Re} \lambda_{\bar{m}}$. Пусть для определенности $1 \leqslant \bar{m} \leqslant n_{1}$.

В работах [5]-[7] в качестве функций $t_{j}(z), 1 \leqslant j \leqslant n_{1}$, рассматривались функции вида $t_{\bar{m}}(z)=t\left(z_{1}, \ldots, z_{\bar{m}}\right)$, определенные как время, за которое точка $z=\left(z_{1}, \ldots, z_{\bar{m}}\right.$, $0, \ldots, 0)$, двигаясь по траектории системы $(28)$, достигнет сферы $\sum_{i=1}^{\bar{m}}\left|z_{i}\right|^{2}=\widetilde{\varepsilon}^{2}$, где $\widetilde{\varepsilon}-$ некоторое положительное число. При этом функции $t_{\bar{m}}(z)$ определены корректно и выполняются все требования относительно гладкости функций (34).

Отметим, что поскольку $\operatorname{Re} \lambda_{\bar{m}+1} \neq \operatorname{Re} \lambda_{\bar{m}}$, то матрища $A$ системы $(28)$ имеет блочно-диагональную структуру $A=\operatorname{diag}\left[\widetilde{A}_{1}, \widetilde{A}_{2}\right]$, где размерность матрищы $\widetilde{A}_{1}$ равна числу $\bar{m}$. Тогда аналогичную структуру имеет и матрица автоморфизма $B=\operatorname{diag}\left[\widetilde{B}_{1}, \widetilde{B}_{2}\right]$, где $\widetilde{B}_{1}$ - матрица размерности $\bar{m}$.

Для наших целей в качестве функций $t_{\bar{m}}(z)$ мы рассмотрим функции, определенные как время, за которое точка $z=\left(z_{1}, \ldots, z_{\bar{m}}, 0, \ldots, 0\right)$ достигнет поверхности

$$
\sum_{i=1}^{\bar{m}} R_{i}(z)=\widetilde{\varepsilon}^{2}, \quad R_{i}(z)=\left\|\widetilde{B}_{1}^{i-1} z\right\|^{2}, \quad 1 \leqslant i \leqslant \bar{m} .
$$

Нетрудно видеть, что функции $t_{\bar{m}}(z)$ определены корректно. Вместе с тем они удовлетворяют всем условиям, которые обеспечивают гладкость функций (34). И, наконец, что самое главное, функции $t_{\bar{m}}(z)$ удовлетворяют условию $t_{\bar{m}}(B z)=t_{\bar{m}}(z)$. Теорема доказана. 
ЗАмЕЧАНИЕ 2. Отметим, что преобразования, построенные при доказательствах утверждений данной работы, обладают тем же свойством, что и преобразования, построенные в [2], а именно: коммутируют не просто с отдельно взятьм автоморфизмом соответствующей системы, а одновременно со всеми линейными автоморфизмами, имеющимися у системы уравнений.

Идею построения функций $h_{1}, h_{2}$ в доказательстве леммы 1 , инвариантных относительно автоморфизма (2), предложил А. Д. Брюно, за что автор выражает ему свою благодарность.

\section{СПИСОК ЦИТИРОВАННОЙ ЛИТЕРАТУРЫ}

[1] Afendikov A., Mielke A. Bifurcation of homoclinic orbits to a saddlefocus in reversible systems with SO(2)-symmetry. Preprint DANCE № 1. Hannover, Germany: University of Hannover, 1998.

[2] Брюно А. Д. Локальный метод нелинейного анализа дифференциальных уравнений. М.: Наука, 1979.

[3] Хартман Ф. Обыкновенные дифференциальные уравнения. М.: Мир, 1970.

[4] Самовол В. С. Эквивалентность систем дифференциальных уравнений в окрестности особой точки // Тр. ММО. 1982. Т. 44. С. 213-234.

[5] Самовол В. С. О необходимом и достаточном условии гладкой линеаризации автономной системы на плоскости в окрестности особой точки // Матем. заметки. 1989. Т. 46. №1. C. $67-77$.

[6] Самовол В. С.Линеаризация систем дифференциальных уравнений в окрестности инвариантных тороидальных многообразий // Тр. ММО. 1979. Т. 38. С. 187-219.

[7] Самовол В.С. Критерий $C^{1}$-гладкой линеаризации автономной системы в окрестности невырожденной особой точки // Матем. заметки. 1991. Т. 49. № 3. С. 91-96.

Российский государственньй гуманитарный университет

Поступило

E-mail: samovol@cityline.ru 16.06 .99 